\title{
Efficacy of disintegrating aspirin in two different models for acute mild-to-moderate pain: sore throat pain and dental pain
}

\author{
Voelker $\mathrm{M}^{1} \cdot$ Schachtel $\mathrm{BP}^{2} \cdot$ Cooper $\mathrm{SA}^{3} \cdot{\text { Gatoulis } \mathrm{SC}^{4}}^{4}$
}

Received: 24 July 2015/ Accepted: 28 October 2015/Published online: 24 November 2015

(C) Springer International Publishing 2015. This article is published with open access at Springerlink.com

\begin{abstract}
A recently developed fast-release aspirin tablet formulation has been evaluated in two different pain models. The dental impaction pain model and the sore throat pain model are widely used for assessing analgesia, including acute mild-to-moderate pain. Both studies were double-blind, randomized, parallel group and compared a single dose of $1000 \mathrm{mg}$ aspirin with $1000 \mathrm{mg}$ paracetamol and with placebo and investigated the onset and overall time course of pain relief. Speed of onset was measured by the double-stopwatch method for time to meaningful pain relief and time to first perceptible pain relief. Pain intensity and pain relief were rated subjectively over a 6-h (dental pain) and 2-h (sore throat pain) time period. In both models fast-release aspirin and commercial paracetamol were statistically significantly different from placebo for onset of action, summed pain intensity differences and total pain relief. Meaningful pain relief was achieved within a median of 42.3 and $42.9 \mathrm{~min}$ for aspirin and paracetamol, respectively, in the dental pain model. The corresponding numbers in sore throat pain were 48.0 and $40.4 \mathrm{~min}$. All treatments in both studies were safe and well tolerated. No serious adverse events were reported and no subject was discontinued due to an adverse event. Overall the two studies clearly demonstrated efficacy over placebo in the two pain models and a comparable efficacy and safety profile between aspirin and an equivalent dose of
\end{abstract}

Voelker M

michael.voelker@bayer.com

Bayer HealthCare, Leverkusen, Germany

2 Schachtel Research Company, Jupiter, FL, USA

3 Clinical Trial Consultant, Palm Beach Gardens, FL, USA

4 Bayer HealthCare, Whippany, NJ, USA paracetamol under the conditions of acute dental pain and acute sore throat pain.

Trial registration These trials were registered with ClinicalTrials.gov, registration number: NCT01420094, registration date: July 27, 2011 and registration number: NCT01453400, registration date: October 13, 2011.

Keywords Aspirin - Acetylsalicylic acid · Paracetamol · Acetaminophen - Onset of action - Dental pain .

Sore throat pain - Acute pain $\cdot$ Pain model $\cdot$ Stopwatch

\section{Introduction}

Aspirin (acetylsalicylic acid, ASA) is a widely used nonsteroidal anti-inflammatory drug (NSAID) with analgesic, antipyretic and anti-inflammatory properties. It is commonly used in the nonprescription setting for the treatment of mild-to-moderate pain (Hersh et al. 2000) and fever (Bachert et al. 2005). Since its introduction several new formulations have been developed and marketed, e.g., granules, effervescent tablets, and chewable tablets. Recently, a fast release, solid tablet formulation has been developed and its efficacy for pain following third molar extraction determined. This new formulation contains sodium carbonate as a disintegrant and small particles of the active ingredient, both contributing to fast dissolution, absorption and consequent onset of action (Voelker and Hammer 2012; Cooper and Voelker 2012).

The fast-release tablet strength is $500 \mathrm{mg}$ of aspirin. Two standard analgesic efficacy studies investigated the onset and overall time course of pain relief compared to an equivalent strength of a conventional non-rapidly disintegrating paracetamol formulation in two different pain models. In these studies two tablets of aspirin $(1000 \mathrm{mg})$ 
were compared with a corresponding dose of paracetamol ( 2 tablets, $1000 \mathrm{mg}$ ). The pain models investigated were dental pain and sore throat pain due to upper respiratory tract infection (URTI). Both models are commonly used for assessing analgesia in acute mild-to-moderate pain (Food and Drug Administration 1988; European Medicine Agency 2002).

The dental impaction pain model has been widely used in the development and assessment of analgesics since the mid-1970s and has several inherently favorable attributes (Cooper and Beaver 1976). In this model, otherwise healthy patients undergo surgical removal of impacted (imbedded in the jaw) third molars. The surgery which involves creating a soft tissue flap, removal of some alveolar bone and sectioning of the impacted tooth or teeth is usually done under local anesthesia with or without short-acting conscious sedation. The subsequent onset of postsurgical pain is predictable and occurs within 1-3 h of the surgery as the effects of the local anesthetic wears off. Patients can be recruited to the study and screened in advance of the elective surgery thus allowing potential confounding treatments to be minimized. The dental impaction pain model is unique amongst the pain models used to study nonprescription analgesics as the time and intensity of pain onset is predictable and other confounding factors are well controlled. In addition, the intensity of the pain resulting from the dental surgical procedure can be prospectively estimated based on which teeth are surgically removed, the number of impacted teeth extracted and the extent (how badly imbedded) of the impactions (Cooper and Desjardins 2010).

The dose-response data generated from studies in the dental impaction pain model also closely parallel the same range of doses that have been evaluated in the sore throat pain model (Schachtel 1991). Like the dental pain model, the sore throat pain model is a tried-and-true analgesic assay (Hersh et al. 2000; European Medicine Agency 2002) used worldwide since the early 1980s (Schachtel et al. 1984) by different investigators (Blagden et al. 2002; Benrimoj et al. 2001). It is based on the most common type of human pain, sore throat, experienced since childhood throughout adulthood. As such, patients are familiar with the condition and find it easy to rate (in terms of pain intensity) and describe (in terms of sensory qualities, in particular, and throat function). As in the dental pain model, subjects in a sore throat pain study tend to be young adults in a university setting; they are excellent "test-takers", adept at documenting responses, and generally otherwise healthy, thus avoiding interference by co-morbid medical conditions or medications that can confound pharmacologic evaluations in a controlled clinical trial. The procedural components of the sore throat pain model (also referred to as the pharyngitis pain model) have been well-described, implementing basic principles of study design, objective confirmation of the pain-producing condition (on the Tonsillo-Pharyngitis Assessment) (Schachtel et al. 2007); specification of homogeneous, well-characterized subjects before treatment, sensitive instruments to rate pain, its relief and functional outcomes (Schachtel et al. 2014a, b). The construct of a sore throat study has been applied to single-dose evaluations, multiple-dose evaluations over $24 \mathrm{~h}$ and 7 days (Blagden et al. 2002; Benrimoj et al. 2001; Schachtel et al. 1988, 2007, 2011, 2014a, b), onset-of-action determinations (Schachtel et al. 1994, 2014a, b), discrimination between doses of the same analgesic (Schachtel et al. 2002, 2010) and between different analgesics (Schachtel et al. 1984) and analgesic adjuvants (Schachtel et al. 1991), revealing the upside assay sensitivity of the model.

\section{Methods}

Both of the reported studies were single-center, randomized, double-blind, double-dummy, placebo-controlled, parallel-group, and assessed the comparative onset of action of the fast-release aspirin tablet in subjects with either postoperative dental pain or sore throat pain. Eligible subjects in either study were randomized in 2:2:1 fashion to either a single dose of aspirin tablets (Aspirin ${ }^{\circledR}$, Bayer HealthCare, Germany) equivalent to $1000 \mathrm{mg}$, paracetamol caplets (Tylenol $^{\circledR}$ Extra Strength, McNeil Consumer Healthcare, US) equivalent to $1000 \mathrm{mg}$, or placebo. The placebos used in the two studies were Aspirin ${ }^{\circledR}$ matching placebo and Tylenol ${ }^{\circledR}$ Extra Strength matching placebo.

The studies were conducted at investigative sites located in the United States. The studies were conducted in accordance with Good Clinical Practice guidance, and each protocol was approved by an institutional review board (IRB). All participants provided written informed consent.

\section{Dental pain}

This study enrolled 510 healthy, ambulatory male and female volunteers, 16-45 years of age, scheduled to undergo surgical extraction of impacted third molars and who had moderate-to-severe postoperative pain.

After experiencing postsurgical pain of at least moderate severity, between approximately 1 and $4 \mathrm{~h}$ after surgery, subjects rated pain intensity (PI) on an 11-point Numerical Pain Intensity Rating Scale (subjects must have reported at least 5 on this scale), a $0-10$ ordinal scale with endpoint of $0=$ no pain and $10=$ very painful, and on a 4 -point Categorical Pain Intensity Scale $(0=$ no pain, $1=$ mild pain, $2=$ moderate pain, $3=$ severe pain).

Eligible subjects were randomly assigned to one of the three treatment groups previously described and were 
administered their assigned dose of study drug. Subjects were included if they were scheduled to under surgical extraction of either two mandibular partial bony impactions or one mandibular full bony alone or in combination with a mandibular partial bony impaction, soft tissue impaction, or erupted third molar. Maxillary third molars could have been removed regardless of impaction level. Subjects were excluded if they had any clinically significant concomitant disease, including asthma, chronic sinusitis, or nasal abnormalities. In addition, subjects were excluded if they had a history of bleeding disorders, including gastrointestinal bleeding or perforation related to previous NSAID therapy. Subjects were also excluded if they had used any analgesics within 5 days of surgery, or any caffeine-containing substances within $12 \mathrm{~h}$ of study drug administration.

Pain intensity and pain relief (PR) were rated by subjects at $5,10,15,20,25,30,35,40,50$, and $60 \mathrm{~min}$ and 1.5, 2, 3, 4,5 , and $6 \mathrm{~h}$ after dosing and immediately prior to the use of any rescue medication. The double-stopwatch method was used to calculate time to "first perceptible PR" and "first perceptible PR confirmed" (stopwatch 1) and time to "meaningful PR" (stopwatch 2). The study coordinator started the 2 stopwatches at the time of dosing.

The primary endpoint was defined as time to meaningful pain relief, while secondary endpoints included:

- time to first perceptible PR,

- time to first perceptible PR confirmed,

- PR and sum of Pain Intensity Difference (PID) scores at $5,10,15,20,25,30,35,40,50$, and $60 \mathrm{~min}$ and at 1.5, 2, 3, 4, 5, and $6 \mathrm{~h}$ after dosing,

- Summed Pain Intensity Differences $\left(\operatorname{SPID}_{0-2}\right)$ (summed, time-weighted PR from 0 to $2 \mathrm{~h}$ after dosing),

- total pain relief (TOTPAR ${ }_{0-2}$ ) (summed, time-weighted total PR from 0 to $2 \mathrm{~h}$ after dosing),

- summary scores for $\mathrm{SPID}_{0-4}$, TOTPAR $0_{0-4}, \mathrm{SPID}_{0-6}$, and TOTPAR $0-6$,

- time to first intake of rescue medication and the cumulative proportion of subjects taking rescue medication by time point,

- global assessment of PR at $6 \mathrm{~h}$ after dosing or immediately before the first intake of rescue medication.

Approximately 500 subjects (200 subjects per active treatment group and 100 subjects for the placebo treatment group) provided $90 \%$ power to detect a treatment difference between aspirin and paracetamol for time to treatment onset at a 2-sided significance level of 0.05 . This sample size was derived under the assumptions that the proportion of subjects who would experience meaningful relief by $6 \mathrm{~h}$ was $76 \%$ for the aspirin group and $60 \%$ for the paracetamol group.

\section{Sore throat pain}

This study enrolled 177 otherwise healthy, ambulatory male and female volunteers, 18 years of age and older who presented to the clinic within 6 days of onset of sore throat pain due to upper respiratory tract infection (URTI). Eligible subjects must have had a baseline sore throat PI score of $\geq 60 \mathrm{~mm}$ on the $100 \mathrm{~mm}$ Sore Throat Pain Intensity Scale (STPIS), and had a score $\geq 5$ on the TonsilloPharyngitis Assessment (TPA).

The TPA is an index that provides descriptions and gradations of the clinical signs of tonsillo-pharyngitis and is based upon previous work by Schachtel et al. (2007). The TPA takes into account ratings (each on a 0-3 point scale) of the intensity of each of seven clinical features of tonsillo-pharyngitis: oral temperature, oropharyngeal color, size of tonsils, number of oropharyngeal exanthemas (vesicles, petechiae or exudates), cervical adenopathy (largest size of anterior cervical lymph nodes), cervical adenopathy (number of anterior cervical lymph nodes) and cervical adenitis (maximum tenderness of some anterior cervical lymph nodes). The sum of these seven ratings comprises the total score for the TPA (i.e., 0-21).

Subjects presented to the clinic within 6 days of onset of sore throat pain due to URTI and were screened for participation in the study. Eligible subjects were stratified by baseline PI ( $\leq 80 \mathrm{vs}>80 \mathrm{~mm}$ ) and randomly assigned in $2: 2: 1$ fashion to one of the three previously described treatment groups and were administered their assigned dose of investigational product. In keeping with its onsetof-action objective, study assessments were conducted over $2 \mathrm{~h}$, Subjects rated their PI at 15, 30, 45, 60, 75, 90, 105 and $120 \mathrm{~min}$ after dosing, and, if applicable, at the time meaningful PR had been achieved and immediately prior to the use of any rescue medication. The double-stopwatch method was used to record analgesic onset of action as described for the dental pain study.

Approximately 175 completing subjects (70 per active treatment group and 35 for the placebo treatment group) provide $80 \%$ power to detect a treatment difference between aspirin and paracetamol for the time to meaningful pain relief at a two-sided significance level of 0.05 . This sample size was derived under the assumption that aspirin would provide approximately a $40 \%$ reduction in the time to meaningful pain relief as compared to paracetamol.

\section{Results}

\section{Dental pain}

The intent-to-treat (ITT) population of study 1 consisted of 510 patients. There were no group differences with respect 
Table 1 Summary of demographics and baseline characteristics

\begin{tabular}{|c|c|c|c|c|}
\hline Study & Variable & Aspirin & Paracetamol & Placebo \\
\hline \multirow[t]{22}{*}{ Dental pain study (study 1) } & ITT population & 204 & 204 & 102 \\
\hline & Age, years [mean $(\mathrm{SD})]$ & $18.2(1.87)$ & $18.2(2.04)$ & $18.2(2.03)$ \\
\hline & Gender ratio, male:female $(\%)$ & $43.1: 56.9$ & $51.5: 48.5$ & 48.0:52.0 \\
\hline & 11-point intensity [mean (SD)] & $7.9(1.31)$ & $7.9(1.30)$ & $7.9(1.27)$ \\
\hline & Categorical pain intensity $(\%)$ & & & \\
\hline & Moderate & 40.7 & 36.3 & 41.2 \\
\hline & Severe & 59.3 & 63.7 & 58.8 \\
\hline & Number of molars removed $(\%)$ & & & \\
\hline & 1 & 4.9 & 3.9 & 6.9 \\
\hline & 2 & 71.6 & 74.0 & 73.5 \\
\hline & 3 & 5.4 & 5.4 & 6.9 \\
\hline & 4 & 18.1 & 16.7 & 12.7 \\
\hline & Tooth sites $(\%)^{\mathrm{a}}$ & & & \\
\hline & Left upper third molar & 51.5 & 51.5 & 35.3 \\
\hline & Left lower third molar & 51.0 & 51.5 & 34.3 \\
\hline & Right upper third molar & 65.7 & 66.2 & 76.5 \\
\hline & Right lower third molar & 68.6 & 66.7 & 79.4 \\
\hline & Impaction score $(\%)^{\mathrm{a}}$ & & & \\
\hline & Erupted in tissue & 2.5 & 2.5 & 0 \\
\hline & Broken soft tissue & 6.4 & 3.9 & 1.0 \\
\hline & Partial bony impaction & 36.3 & 41.7 & 40.2 \\
\hline & Full bony impaction & 92.2 & 90.2 & 94.1 \\
\hline \multirow[t]{9}{*}{ Sore throat pain study (study 2) } & ITT population & 71 & 70 & 36 \\
\hline & Age, years [mean $(\mathrm{SD})]$ & $19.5(1.77)$ & $19.4(1.44)$ & $19.7(1.22)$ \\
\hline & Gender ratio, male:female $(\%)$ & $57.7: 42.3$ & $45.7: 54.3$ & $50.0: 50.0$ \\
\hline & Pain intensity score on $100 \mathrm{~mm}$ VAS [mean (SD)] & $75.1(8.28)$ & $75.9(8.37)$ & $74.1(9.83)$ \\
\hline & Pain intensity $(\%)$ & & & \\
\hline & Greater than $80 \mathrm{~mm}$ & 26.8 & 25.7 & 27.8 \\
\hline & Less than or equal to $80 \mathrm{~mm}$ & 73.2 & 74.3 & 72.2 \\
\hline & Days having sore throat [mean (SD)] & $2.6(1.31)$ & $2.7(1.14)$ & $2.5(1.03)$ \\
\hline & Tonsillo-pharyngitis assessment [mean (SD)] & $9.2(2.95)$ & $10.1(2.94)$ & $9.8(2.97)$ \\
\hline
\end{tabular}

${ }^{a}$ Patients may had more than 1 affected tooth site or impaction score

to age, gender and baseline pain intensity (Table 1). The average age of the subjects was approximately 18 years. About $60 \%$ of subjects on average had severe pain and about $40 \%$ moderate pain at baseline. Mean pain intensity on the 11-point pain intensity scale was 7.9 at baseline. Two molars had been removed in more than $70 \%$ of subjects and almost all subjects had a full bony impaction $(>90 \%)$.

The Kaplan-Meier curve of the primary efficacy endpoint, time to meaningful pain relief, showed statistically both the aspirin group and the paracetamol group separated from the placebo group $(p<0.001)$ (Fig. 1; Table 2). Descriptively there was no difference between the two active groups $(p=0.945)$. A single dose of either $1000 \mathrm{mg}$ aspirin or $1000 \mathrm{mg}$ paracetamol achieved meaningful pain relief within a median of 42.3 and $42.9 \mathrm{~min}$, respectively. For placebo, no median time to meaningful pain relief could be calculated due to the number of censored observations. Median time to first perceptible pain relief was obtained within $17.2 \mathrm{~min}$ for aspirin, $15.0 \mathrm{~min}$ for paracetamol and $27.3 \mathrm{~min}$ for placebo. Differences for active vs placebo were statistically significant $(p<0.001)$; whereas, differences between actives were not $(p=0.392)$.

The SPID and TOTPAR results were consistent overall. SPID and TOTPAR at $2 \mathrm{~h}$ were comparable between aspirin and paracetamol and both were statistically superior to placebo $(p<0.001)$. At 4 and $6 \mathrm{~h}$ SPID and TOTPAR for both actives were statistically significantly different 
Fig. 1 Dental pain study (study 1) Kaplan-Meier plot of time to meaningful pain relief. Green curve aspirin, red curve paracetamol, blue curve placebo

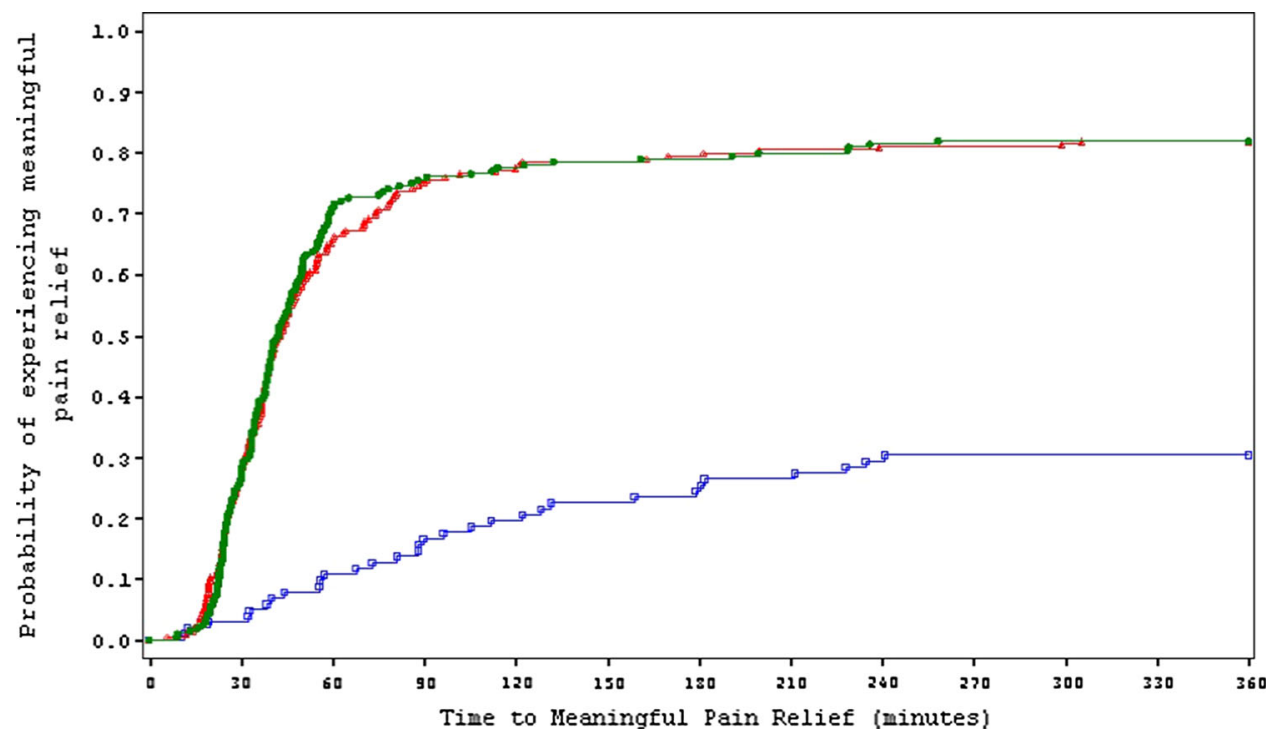

from placebo (all $p<0.001$ ). There were statistically significant differences between aspirin and paracetamol showing a non-confirmatory trend favoring paracetamol $(p=0.002$ and $<0.001$ for SPID 4 and $6 \mathrm{~h} ; p<0.001$ for TOTPAR 4 and $6 \mathrm{~h}$ ).

Both active treatments were safe and well tolerated. No serious adverse events occurred and no subject was discontinued due to an adverse event.

\section{Sore throat pain}

One hundred and seventy-seven subjects were treated when conducting the sore throat study (study 2). Demographic and baseline characteristics were similar across the 3 treatment groups (Table 1). The overall mean age was 19.5 years. At baseline, the overall mean pain intensity score was $75.2 \mathrm{~mm}$, and most subjects (about $73 \%$ ) had a baseline pain intensity score less than or equal to 80 (moderate pain). The baseline upper respiratory tract infection symptoms reported most frequently included sore throat $(100.0 \%)$, swollen neck glands $(78.0 \%)$, tender neck glands $(76.8 \%)$, drowsiness and lack of energy (53.1\% each), and headache (51.4\%), and were reported by a similar number of subjects across the three treatment groups. Subjects had a sore throat for an overall mean of 2.6 days at study entry and a mean tonsillo-pharyngitis score of 9.6 .

The primary efficacy endpoint was time to meaningful pain relief. The corresponding Kaplan-Meier figure is presented in Fig. 2. Mean time to meaningful pain relief was $48.0 \mathrm{~min}$ for aspirin, $40.4 \mathrm{~min}$ for paracetamol and was not achieved for placebo within the observation period of $2 \mathrm{~h}$ (Table 2). Differences to placebo were statistically significant $(p<0.001)$; whereas, the difference between aspirin and paracetamol was not $(p=0.772)$. For time to first perceptible pain relief, differences to placebo were significant $(p<0.001)$; whereas, differences between aspirin and paracetamol were not significant $(p=0.523)$ (aspirin $33.3 \mathrm{~min}$, paracetamol $30.5 \mathrm{~min}, \quad$ placebo 90.8 min) (Table 2).

Summed pain intensity differences measured as SPID0$1 \mathrm{~h}$ and SPID0-2h showed consistently significant differences between aspirin and placebo $(p<0.001)$ and between paracetamol and placebo $(p<0.001)$. Differences between aspirin and paracetamol were not significant ( $p=0.632$ and 0.869 , respectively) (Table 2).

The treatments were safe and well tolerated. No serious adverse events were reported and no subject was discontinued due to an adverse event.

\section{Safety and tolerability}

Adverse events reported in the two studies are shown in Table 3. In the dental pain study, the percentages of subjects with at least one adverse event were 9.8, 10.8 and $17.6 \%$ for aspirin, paracetamol and placebo, respectively. The corresponding numbers in the sore throat study were $18.3,14.3$ and $33.3 \%$, respectively. The numbers of adverse events reported for the gastrointestinal system organ class were $7.4 \%$ (aspirin), $8.3 \%$ (paracetamol) and $9.8 \%$ (placebo) and $4.2 \%$ (aspirin), $4.3 \%$ (paracetamol) and $8.3 \%$ (placebo). 
Table 2 Time to relief and efficacy results

\begin{tabular}{|c|c|c|c|c|}
\hline Study & Variable & Aspirin & Paracetamol & Placebo \\
\hline \multirow[t]{28}{*}{ Dental pain study (study 1) } & Median time to FPR, $\min (95 \% \mathrm{CI})$ & $17.2(15.0,19.1)$ & $15.0(14.6,16.1)$ & $27.3(20.0,35.0)$ \\
\hline & $p$ value vs aspirin & - & 0.392 & $<0.001$ \\
\hline & $p$ value vs paracetamol & - & - & $<0.001$ \\
\hline & $\%$ of subjects achieved FPR & 96.6 & 97.1 & 70.6 \\
\hline & $\%$ of subjects censored & 3.4 & 2.9 & 29.4 \\
\hline & Median time to $\mathrm{MR}, \min (95 \% \mathrm{CI})$ & $42.3(38.8,46.5)$ & $42.9(38.8,48.2)$ & NA (NA, NA) \\
\hline & $p$ value vs aspirin & - & 0.945 & $<0.001$ \\
\hline & $p$ value vs paracetamol & - & - & $<0.001$ \\
\hline & $\%$ of subjects achieved MR & 81.9 & 81.9 & 30.4 \\
\hline & $\%$ of subjects censored & 18.1 & 18.1 & 69.6 \\
\hline & SPID 0-2 h, mean (SD) & $2.4(1.5)$ & $2.6(1.4)$ & $0.3(1.3)$ \\
\hline & $p$ value vs aspirin & - & 0.267 & $<0.001$ \\
\hline & $p$ value vs paracetamol & - & - & $<0.001$ \\
\hline & SPID 0-4 h, mean (SD) & $4.4(3.3)$ & $5.4(3.2)$ & $0.8(3.3)$ \\
\hline & $p$ value vs aspirin & - & 0.002 & $<0.001$ \\
\hline & $p$ value vs paracetamol & - & - & $<0.001$ \\
\hline & SPID 0-6 h, mean (SD) & $5.9(5.1)$ & $7.9(4.9)$ & $1.3(5.5)$ \\
\hline & $p$ value vs aspirin & - & $<0.001$ & $<0.001$ \\
\hline & $p$ value vs paracetamol & - & - & $<0.001$ \\
\hline & TOTPAR 0-2 h, mean (SD) & $4.3(1.9)$ & $4.5(1.8)$ & $1.4(1.6)$ \\
\hline & $p$ value vs aspirin & - & 0.283 & $<0.001$ \\
\hline & $p$ value vs paracetamol & - & - & $<0.001$ \\
\hline & TOTPAR 0-4 h, mean (SD) & $8.0(4.4)$ & $9.5(4.2)$ & $3.0(4.1)$ \\
\hline & $p$ value vs aspirin & - & $<0.001$ & $<0.001$ \\
\hline & $p$ value vs paracetamol & - & - & $<0.001$ \\
\hline & TOTPAR 0-6 h, mean (SD) & $11.0(7.0)$ & $14.0(6.7)$ & $4.5(6.8)$ \\
\hline & $p$ value vs aspirin & - & $<0.001$ & $<0.001$ \\
\hline & $p$ value vs paracetamol & - & - & $<0.001$ \\
\hline \multirow[t]{16}{*}{ Sore throat pain study (study 2) } & Median time to FPR, $\min (95 \% \mathrm{CI})$ & $33.3(28.9,41.7)$ & $30.5(25.7,35.1)$ & $90.8(45.8, \mathrm{NA})$ \\
\hline & $p$ value vs aspirin & - & 0.523 & $<0.001$ \\
\hline & $p$ value vs paracetamol & - & - & $<0.001$ \\
\hline & $\%$ of subjects achieved PR & 91.5 & 91.3 & 52.8 \\
\hline & $\%$ of subjects censored & 8.5 & 8.7 & 47.2 \\
\hline & Median time to $\mathrm{MR}, \min (95 \% \mathrm{CI})$ & $48.0(39.7,56.9)$ & $40.4(35.3,53.7)$ & NA (NA, NA) \\
\hline & $p$ value vs aspirin & - & 0.772 & $<0.001$ \\
\hline & $p$ value vs paracetamol & - & - & $<0.001$ \\
\hline & $\%$ of subjects achieved MR & 73.2 & 79.7 & 30.6 \\
\hline & $\%$ of subjects censored & 26.8 & 20.3 & 69.4 \\
\hline & SPID 0-1 h, mean (SD) & $15.0(12.6)$ & $16.1(14.6)$ & $4.2(8.6)$ \\
\hline & $p$ value vs aspirin & - & 0.632 & $<0.001$ \\
\hline & $p$ value vs paracetamol & - & - & $<0.001$ \\
\hline & SPID 0-2 h, mean (SD) & $48.0(33.3)$ & $47.1(3.4)$ & $13.4(22.0)$ \\
\hline & $p$ value vs aspirin & - & 0.869 & $<0.001$ \\
\hline & $p$ value vs paracetamol & - & - & $<0.001$ \\
\hline
\end{tabular}

$F P R$ first perceptible pain relief, $M R$ meaningful relief, $C I$ confidence interval, SPID summed pain intensity differences, TOTPAR total pain relief, $N A$ not available 
Fig. 2 Sore throat pain study (study 2) Kaplan-Meier plot of time to meaningful pain relief. Green curve aspirin, red curve paracetamol, blue curve placebo

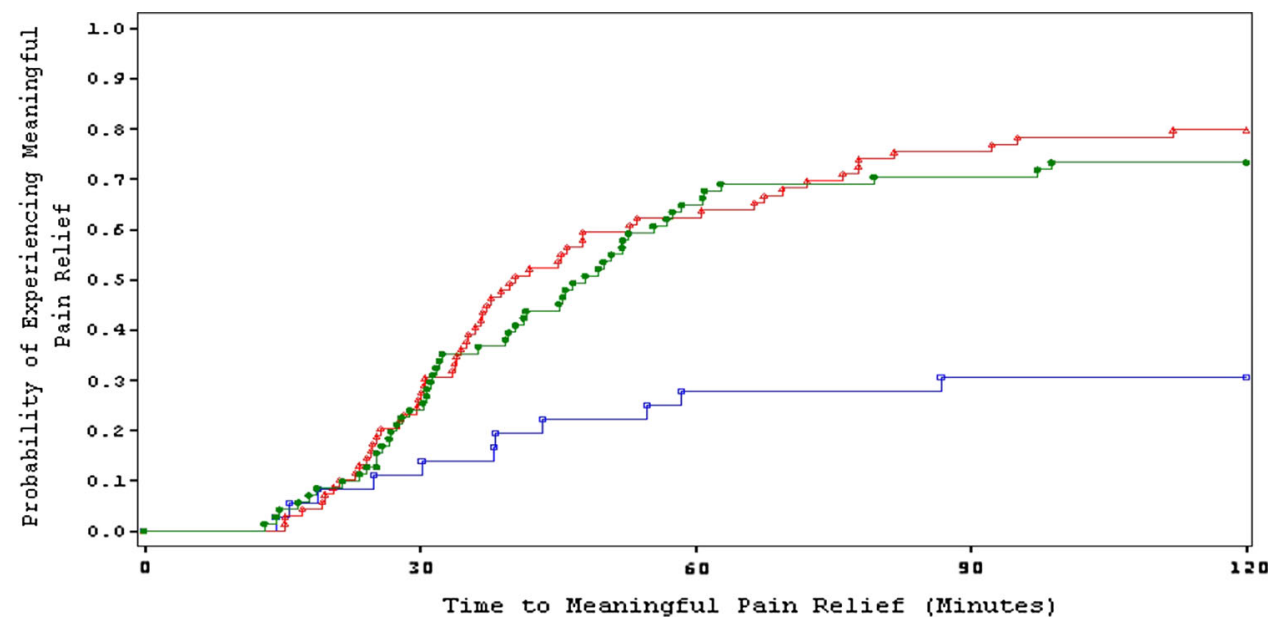

Table 3 Treatment-emergent adverse events (AE) reported by at least $1 \%$ of patients overall (safety population)

\begin{tabular}{|c|c|c|c|c|}
\hline Study & Variable & Aspirin & Paracetamol & Placebo \\
\hline \multirow[t]{9}{*}{ Dental pain study (study 1) } & Number of patients & 204 & 204 & 102 \\
\hline & Total number of AEs & 30 & 36 & 30 \\
\hline & Number of subjects with at least $1 \mathrm{AE}[n(\%)]$ & $20(9.8)$ & $22(10.8)$ & $18(17.6)$ \\
\hline & Gastrointestinal disorders & $15(7.4)$ & $17(8.3)$ & $10(9.8)$ \\
\hline & Nausea & $11(5.4)$ & $16(7.8)$ & $10(9.8)$ \\
\hline & Vomiting & $8(3.9)$ & $9(4.4)$ & $4(3.9)$ \\
\hline & Nervous system disorder & $4(2.0)$ & $4(2.0)$ & $7(6.9)$ \\
\hline & Headache & $4(2.0)$ & $2(1.0)$ & $3(2.9)$ \\
\hline & Dizziness & 0 & $1(0.5)$ & $4(3.9)$ \\
\hline \multirow[t]{21}{*}{ Sore throat pain study (study 2) } & Number of patients & 71 & 70 & 36 \\
\hline & Total number of AEs & 20 & 11 & 13 \\
\hline & Number of subjects with at least $1 \mathrm{AE}[n(\%)]$ & $13(18.3)$ & $10(14.3)$ & $12(33.3)$ \\
\hline & Gastrointestinal disorders & $3(4.2)$ & $3(4.3)$ & $3(8.3)$ \\
\hline & Nausea & $1(1.4)$ & $1(1.4)$ & $1(2.8)$ \\
\hline & Vomiting & $2(2.8)$ & 0 & 0 \\
\hline & Enlarged uvula & $1(1.4)$ & 0 & $1(2.8)$ \\
\hline & Respiratory, thoracic, and mediastinal disorders & & & \\
\hline & Oropharyngeal pain & $7(9.9)$ & $2(2.9)$ & $5(13.9)$ \\
\hline & Cough & $3(4.2)$ & $1(1.4)$ & $2(5.6)$ \\
\hline & Nasal congestion & $1(1.4)$ & 0 & $2(5.6)$ \\
\hline & Wheezing & $1(1.4)$ & $1(1.4)$ & $1(2.8)$ \\
\hline & & $3(4.2)$ & 0 & 0 \\
\hline & Infections and infestations & $4(5.6)$ & $1(1.4)$ & $4(11.1)$ \\
\hline & Laryngitis & $1(1.4)$ & 0 & $2(5.6)$ \\
\hline & Upper respiratory tract infection & $3(4.2)$ & 0 & 0 \\
\hline & Tonsillitis & 0 & $1(1.4)$ & $1(2.8)$ \\
\hline & General disorders and administration site conditions & $2(2.8)$ & 0 & $1(2.8)$ \\
\hline & Pain & $2(2.8)$ & 0 & 0 \\
\hline & Nervous system disorders & $1(1.4)$ & $2(2.9)$ & 0 \\
\hline & Headache & $1(1.4)$ & $1(1.4)$ & 0 \\
\hline
\end{tabular}




\section{Discussion}

For the treatment of acute mild-to-moderate pain, patients seek fast onset of relief (Hersh et al. 2000; Schachtel et al. 1999). Recently a fast release aspirin formulation, characterized by the inclusion of sodium carbonate as a disintegrant and smaller active ingredient particles, has been developed and approved in many countries for the treatment of mild-to-moderate acute pain. This formulation has improved dissolution and pharmacokinetics and consequently faster onset of action compared to the original aspirin formulation (Voelker and Hammer 2012; Cooper and Voelker 2012). The time to meaningful pain relief is an important clinical endpoint providing a very relevant advantage for patients with mild-to-moderate pain (Schachtel et al. 1999). In the recent dental pain study (Cooper and Voelker 2012), a time of $49.4 \mathrm{~min}$ for the fastrelease formulation compared to $99.2 \mathrm{~min}$ for the original formulation has been determined, indicating a twofold improvement in analgesic onset. However, it is important to provide evidence that this endpoint can be achieved in different pain models. In addition to acute dental pain, the sore throat pain model is also well-regarded by researchers and regulators as an appropriate general pain model (Hersh et al. 2000; European Medicine Agency 2002). Furthermore, relative performance of analgesic active ingredients and analgesic medicinal products is of high interest with respect to decision making in analgesia.

The two present studies confirm the results seen in the earlier study (Cooper and Voelker 2012). Time to meaningful pain relief for fast-release aspirin was determined to be $42.3 \mathrm{~min}$ in dental pain. Small differences to the results seen in the earlier study (Cooper and Voelker 2012) may be explained by study and population variability. Also in the study with patients having sore throat pain and treated with the fast-disintegrating aspirin, the time to meaningful pain relief (48 min) is consistent with the dental pain study. Generally, the results of the present dental pain and sore throat pain studies confirm the data of the earlier dental pain study (Cooper and Voelker 2012). Neither the dental pain study nor the sore throat pain study showed a statistically significant difference between aspirin $1000 \mathrm{mg}$ and paracetamol $1000 \mathrm{mg}$ in any determination of onset.

Conventional aspirin is absorbed in the stomach and in the upper small intestine, but the upper small intestine is considered to be the main absorption site because of the poor solubility of aspirin in the acidic $\mathrm{pH}$ of the stomach and the small absorption surface of the stomach mucosa, as opposed to the much greater surface of the small intestine (Schroer 2009). The new fast-disintegrating formulation is characterized by its small particle size and the sodium carbonate disintegrant. This theoretically supports absorption in the stomach by enhancing disintegration of the tablet as well as increasing surface area due to the smaller particle size. In addition, the new aspirin formulation may accelerate gastric emptying. This factor may be clinically important since sympathetic stimulation due to pain or its associated trauma may slow gastric emptying (Jamali and Kunz-Dober 1999; Jamali and AghazadehHabashi 2008). Interestingly, the time to maximum plasma concentration $\left(T_{\max }\right)$ for the new aspirin formulation is comparable to soluble highly buffered aspirin formulations (Voelker and Hammer 2012; Kanani et al. 2015) which also may enhance gastric emptying. However, this is very speculative and needs to be more intensively investigated.

\section{Conclusions}

The two efficacy studies described in this paper further support the efficacy and safety of the novel fast-release aspirin formulation. This formulation is characterized by the inclusion of sodium carbonate as a disintegrant and smaller active ingredient particles. Efficacy in two different acute pain models has been shown. Patients with dental pain and patients with sore throat pain showed significantly faster and better pain relief when treated with aspirin compared to patients treated with placebo. Generally, the two studies demonstrated an equivalent efficacy and safety profile for equivalent doses of aspirin and paracetamol $(1000 \mathrm{mg}$ ) under the conditions of acute dental pain and acute sore throat pain.

Acknowledgments Declaration of interest: The studies were sponsored by Bayer HealthCare. B.S. and S.C. acted as consultants for Bayer HealthCare and received consultancy fees from Bayer HealthCare for their support in conducting the studies. B.S. acted as principle investigator for Bayer HealthCare sponsored studies and received investigator fees from Bayer HealthCare. B.S. and S.C. were not compensated for their support of this publication. M.V. and S.G. are employees of Bayer HealthCare.

Open Access This article is distributed under the terms of the Creative Commons Attribution 4.0 International License (http:// creativecommons.org/licenses/by/4.0/), which permits unrestricted use, distribution, and reproduction in any medium, provided you give appropriate credit to the original author(s) and the source, provide a link to the Creative Commons license, and indicate if changes were made.

\section{References}

Bachert C, Chuchalin AG, Eisebitt et al (2005) Aspirin compared with acetaminophen in the treatment of fever and other symptoms of upper respiratory tract infection in adults: a multicenter, randomized, double-blind, double-dummy, placebo 
controlled, parallel-group, single-dose, 6-hour dose-ranging study. Clin Ther 27:993-1003

Benrimoj SJ, Langford JH, Christian J et al (2001) Efficacy and tolerability of the anti-inflammatory throat lozenge flurbiprofen $8.75 \mathrm{mg}$ in the treatment of sore throat. A randomized doubleblind, placebo-controlled study. Clin Drug Invest 21:183-193

Blagden M, Christian J, Miller K et al (2002) A. Multidose flurbiprofen $8.75 \mathrm{mg}$ lozenges in the treatment of sore throat: a randomized, double-blind, placebo-controlled study in UK general practice centers. Int J Clin Pract 56:95-100

Cooper SA, Beaver WT (1976) A model to evaluate mild analgesics in oral surgery out-patients. Clin Pharmacol Ther 20:241-250

Cooper SA, Desjardins PJ (2010) The value of the dental impaction pain model in drug development. In: Szallasi A (ed) Analgesia, methods in molecular biologies series. The Humana Press, New York, pp 175-190

Cooper SA, Voelker M (2012) Evaluation of onset of pain relief from micronized aspirin in dental pain model. Inflammapharmacology 20:233-242

European Medicine Agency (2002) Committee for proprietary medicinal products. Note for guidance on clinical investigations of medicinal products for treatment of nociceptive pain. London, 21 November 2002 CPMP/EWP/612/00

Food and drug administration (1988) Internal Analgesics TFM 11/16/ 1988. Vol. 74, No. 221 [Docket No. 77N-0094]

Hersh EV, Moore PA, Ross GL (2000) Over-the-counter analgesics and antipyretics: a critical assessment. Clin Ther 22:500-548

Jamali F, Aghazadeh-Habashi A (2008) Rapidly dissolving formulations for quick absorption during pain episodes: ibuprofen. Int $\mathrm{J}$ Clin Pharmacol Ther 46:55-63

Jamali F, Kunz-Dober CM (1999) Pain-mediated altered absorption and metabolism of ibuprofen: an explanation for decreased serum enantiomer concentration after dental surgery. Br J Clin Pharmacol 47:391-396

Kanani K, Gatoulis S, Voelker M (2015) Influence of differing analgesic formulations of aspirin on pharmacokinetic parameters. Pharmaceutics 7:188-198

Schachtel BP (1991) Sore throat pain. In: Max MB, Portenoy RK, Laska EM (eds) The design of analgesic clinical trials (advances in Pain Research and Therapy: vol 18). Raven Press, New York, pp 393-406
Schachtel BP, Fillingim JM, Beiter DJ et al (1984) Rating scales for analgesics in sore throat. Clin Pharmacol Ther 36:151-156

Schachtel BP, Fillingim JM, Thoden WR et al (1988) Sore throat pain in the evaluation of mild analgesics. Clin Pharmacol Ther 44:704-711

Schachtel BP, Fillingim JM, Lane AC et al (1991) Caffeine as an analgesic adjuvant. A double-blind study comparing aspirin with caffeine to aspirin and placebo in patients with sore throat. Arch Intern Med 151:733-737

Schachtel BP, Cleves GS, Konerman JP et al (1994) A placebocontrolled model to assay the onset of nonprescription-strength analgesic drugs. Clin Pharmacol Ther 55:464-470

Schachtel BP, Loose I, Brown AT et al (1999) Onset of action defined by patients. Clin Pharmacol Ther 65:117

Schachtel BP, Homan HD, Gibb IA et al (2002) Demonstration of dose response of flurbiprofen lozenges with the sore throat pain model. Clin Pharmacol Ther 71:375-380

Schachtel BP, Pan S, Kohles JD et al (2007) Utility and sensitivity of the sore throat pain model: results of a randomized controlled trial on the COX-2 selective inhibitor valdecoxib. J Clin Pharmacol 47:860-870

Schachtel BP, Voelker M, Sanner KM et al (2010) Demonstration of the analgesic efficacy and dose-response of acetylsalicylic acid with pseudoephedrine. J Clin Pharmacol 50:1429-1437

Schachtel BP, McCabe D, Berger M, Zhang R, Sanner KM, Savino L, Rizouk J, Schachtel EP (2011) Efficacy of low-dose celecoxib in patients with acute pain. J Pain 12:756-763

Schachtel B, Aspley S, Shephard A et al (2014a) Utility of the sore throat pain model in a multiple-dose assessment of the acute analgesic flurbiprofen: a randomized controlled study. Trials 15:263-270

Schachtel B, Aspley S, Shephard A et al (2014b) Onset of action of a lozenge containing flurbiprofen $8.75 \mathrm{mg}$ : a randomized, doubleblind, placebo-controlled trial with a new method for measuring onset of analgesic activity. Pain 155:422-428

Schroer K Acetylsalicylic Acid (2009) WILEY-VCH Verlag, Weinheim, Germany

Voelker M, Hammer M (2012) Dissolution and pharmacokinetics of a novel micronized aspirin formulation. Inflammopharmacology 20:225-231 